\title{
Research status and prospects of biomarkers for nasopharyngeal carcinoma in the era of high-throughput omics (Review)
}

\author{
SHAN-QIANG ZHANG ${ }^{1}$, SU-MING PAN ${ }^{2}$, SI-XIAN LIANG ${ }^{2}$, YU-SHUAI HAN ${ }^{3}$, \\ HAI-BIN CHEN ${ }^{4}$ and JI-CHENG LI ${ }^{1,3}$

\begin{abstract}
${ }^{1}$ Medical Research Center and ${ }^{2}$ Department of Radiotherapy, Yue Bei People's Hospital, Shantou University Medical College, Wujiang, Shaoguan, Guangdong 512025; ${ }^{3}$ Institute of Cell Biology, Zhejiang University School of Medicine,

Hangzhou, Zhejiang 310058; ${ }^{4}$ Department of Histology and Embryology,

Shantou University Medical College, Shantou, Guangdong 515041, P.R. China
\end{abstract}

Received June 24, 2020; Accepted January 21, 2021

DOI: $10.3892 /$ ijo.2021.5188

\begin{abstract}
As a malignant tumor type, nasopharyngeal carcinoma (NPC) is characterized by distinct geographical, ethnic and genetic differences; presenting a major threat to human health in many countries, especially in Southern China. At present, no accurate and effective methods are available for the early diagnosis, efficacious evaluation or prognosis prediction for NPC. As such, a large number of patients have locoregionally advanced NPC at the time of initial diagnosis. Many patients show toxic reactions to overtreatment and have risks of cancer recurrence and distant metastasis owing to insufficient treatment. To solve these clinical problems, high-throughput '-omics' technologies are being used to screen and identify specific molecular biomarkers for NPC. Because of the lack of comprehensive descriptions regarding NPC biomarkers, the present study summarized the research progress that has been made in recent years to discover NPC biomarkers, highlighting the existing problems that require exploration. In view of the lack of authoritative reports at present, study design factors that affect the screening of biomarkers are also discussed here and prospects for future research are proposed to provide references for follow-up studies of NPC biomarkers.
\end{abstract}

\section{Contents}

1. Introduction

2. NPC biomarkers discovered through genomics

3. NPC biomarkers discovered through transcriptomics

Correspondence to: Professor Ji-Cheng Li, Medical Research Center, Yue Bei People's Hospital, Shantou University Medical College, 133 Huimin South Road, Wujiang, Shaoguan, Guangdong 512025, P.R. China

E-mail: lijichen@zju.edu.cn

Key words: nasopharyngeal carcinoma, omics, high throughput, biomarker
4. NPC biomarkers discovered through proteomics

5. NPC biomarkers discovered through metabolomics

6. Key points for study design

7. Conclusion and future perspectives

\section{Introduction}

As a type of malignant head and neck tumor, nasopharyngeal carcinoma (NPC) is characterized by early extensive local infiltration, high hematogenous dissemination, early lymphatic spread and a high mortality rate (1). According to the International Agency for Research on Cancer (IARC), there were an estimated 129,079 new cases of NPC and 72,987 NPC-related deaths in 2018 worldwide (2). While this is only a small proportion of all cancers (the number of NPC cases in 2018 accounted for $0.7 \%$ of all confirmed cancers), in some high-risk countries or regions, many patients present with NPC as well as drug toxicity due to anti-NPC treatment. China has the highest incidence rate of NPC worldwide. In 2018, there were 60,558 new NPC cases (47.7\% of total global cases) and 31,413 NPC-related deaths in China $(1,2)$. Within China, the incidence rate of NPC is highest in the Guangdong Province; hence, NPC is also commonly referred to as 'Cantonese cancer' (3). NPC has a high incidence rate in other provinces of China, such as Guangxi, Hunan and Fujian, as well as in other countries, such as Indonesia, Vietnam, India, Morocco, Algeria, Tunisia and Ghana (4). Therefore, the prevention and treatment of NPC is an important goal for medical researchers.

To date, the pathogeny behind NPC remains unclear. Existing evidence shows that heredity, race, environment, diet, habits and the Epstein-Barr virus (EBV) are closely related to the pathogenesis behind NPC (1). However, the study showed that NPC development involves a complex interaction of multiple factors, as demonstrated by some gene polymorphisms with different genetic characteristics that are carried by ethnic groups in high-risk areas being associated with different degrees of NPC risk as well as the processing and presentation of EBV antigens. Extracts of Cantonese salted fish from China, as well as herbal medicines used by Chinese and Naga people, have been shown to promote the activation and 
proliferation of EBV, leading to the occurrence of NPC (5). In addition, EBV inducers are detected in soil extracts from NPC-endemic areas in southern China as well as in some vegetables grown in these soils (6). These factors attribute for the increased NPC incidence rate observed in specific regions or races. However, how the genetic factors of different races affect tumor development still requires more systematic and comprehensive research.

Effective early diagnosis and treatment are key to preventing the progression, recurrence and metastasis of NPC. Based on the guidelines published by the World Health Organization (WHO), pathological evidence found by biopsies is the gold standard for NPC diagnosis (7-9). However, because of poor compliance of patients with this invasive procedure, the fact that the symptoms of NPC are not conclusive at early stages and that NPC is not conducive to imaging screening, more than $70 \%$ of patients have locoregionally advanced (LA)-NPC at the time of initial diagnosis (10). Because the location of the NPC is close to the base of the skull and its shape is irregular, it is difficult to distinguish the tumors from nasopharyngeal lymphoma in the early stage using MRI or computed tomography scanning (11). Furthermore, imaging-dependent tumor staging is initially established based on patient survival statistics and is often affected by the subjective judgement of the clinician; therefore, a reliable laboratory method is needed to assist in accurate NPC diagnosis $(4,12)$.

The treatment decision for NPC at different stages is made based on the tumor-node-metastasis (TNM) staging system developed by the Union for International Cancer Control/American Joint Committee on Cancer and the National Comprehensive Cancer Network (NCCN). Radiotherapy (RT) alone is the standard treatment for early NPC, while for patients with stages III-IVa, induction chemotherapy (IC) followed by concurrent chemoradiotherapy (CCRT) or CCRT followed by adjuvant chemotherapy (AC) are the preferred category $2 \mathrm{~A}$ treatment regimen $(4,12)$. A number of clinical trials have investigated the effectiveness of these treatment methods (10,13-15). Although the 5-year overall survival (OS), progression-free survival (PFS), distant metastasis-free survival (DMFS) and locoregional relapse-free survival (LRFS) are improved to various degrees by the application of RT or the one of the various chemotherapy (CT) regimens, there are still many controversies in the clinical application of these treatment methods because of the lack of accurate efficacy evaluation indicators. For example: i) Some patients still have tumor recurrence or distant metastasis after receiving radical RT or IC+CCRT/CCRT+AC, indicating that a more effective treatment should be developed (16-19); ii) IC+CCRT improves the OS and DMFS of patients with LA-NPC to a limited degree, but most patients do not benefit from it, suggesting overtreatment (13-15); iii) patients have poor tolerance to toxic side effects of the treatment, which could lead to delays or interruptions in treatment, thus increasing the risk of tumor progression or drug resistance (1,20-22); and iv) the clinical use of Response Evaluation Criteria in Solid Tumors (RECIST) can only provide a reference for the NPC treatment effect evaluation through generalized remission or progression, and cannot provide quantifiable prediction indexes for NPC recurrence and metastasis. Moreover, the prognosis model based on the TNM staging system cannot provide an objective assessment for the risk of disease progression for patients with NPC $(23,24)$. These problems indicate issues regarding NPC diagnosis, treatment and prognosis, and suggest the need for individualized treatment. Therefore, researchers are focusing on the identification of molecular biomarkers to establish new non-invasive early diagnostic methods, ideal treatment regimens, efficacy evaluation standards and prognostic indicators for NPC (Fig. 1).

Biomarkers can be divided into two categories according to their functions: Those used to discover the molecular mechanisms of action of the disease and drug targets, and those used for the prediction, early diagnosis, efficacy evaluation and prognosis. Several review articles have summarized both types of NPC biomarkers (7-9,25). With the development of high-throughput omics technologies in recent years, many novel biomarkers have been discovered. In the present review, studies on bodily fluid samples, such as serum, plasma and saliva of patients with NPC in the past 5 years were summarized, and the research status of biomarkers for the early diagnosis, treatment (such as RT and $\mathrm{CT}$ ) and prognosis (such as metastasis and recurrence) of NPC were reviewed at the aspects of genomics, transcriptomics, proteomics and metabolomics. The intersection points (such as TNM staging, therapeutic method, sample type and high-throughput technology) in the study design of the existing research and the development trends of NPC biomarkers in the future were also discussed.

\section{NPC biomarkers discovered through genomics}

Genomics aims to collectively characterize and quantify all genes in an organism to study the influence of their interactions (26). It uses high-throughput DNA sequencing, bioinformatics, genetic analysis and functional identification to analyze the structure and function of the whole genome, which forms the omics framework of systems biology with transcriptomics, proteomics and metabolomics (27). In the study of biomarkers, gene mutations are often difficult to match with corresponding clinical phenotypes of the disease. Therefore, researchers must carry out repeated validation studies in large cohorts or conduct supplementary studies with other omics technologies (28).

Based on the fact that genetics, environment, diet and EBV are generally considered as risk factors of cancer, exploring new pathogenic genes and mechanisms to assess the risk of NPC in high-risk population has become the goal of genomics research $(29,30)$. With the wide application of high-throughput technologies, such as whole-exome sequencing, whole-genome sequencing (WGS) and genome-wide association studies (GWAS), many single nucleotide polymorphisms (SNPs) associated with NPC have been identified and comprehensively described (1,7,30-32). However, although the interactions between multiple genes make the complex mechanism of action behind NPC more difficult to understand, simple research design and mature detection technology still push the research forward (33). In recent years, researchers have identified the association between NPC susceptibility and the SNPs of genes encoding for major histocompatibility complex II (34), monocyte chemoattractant protein-1 promoter (35), xeroderma pigmentosum group $\mathrm{D}$, human 8-oxoguanine 

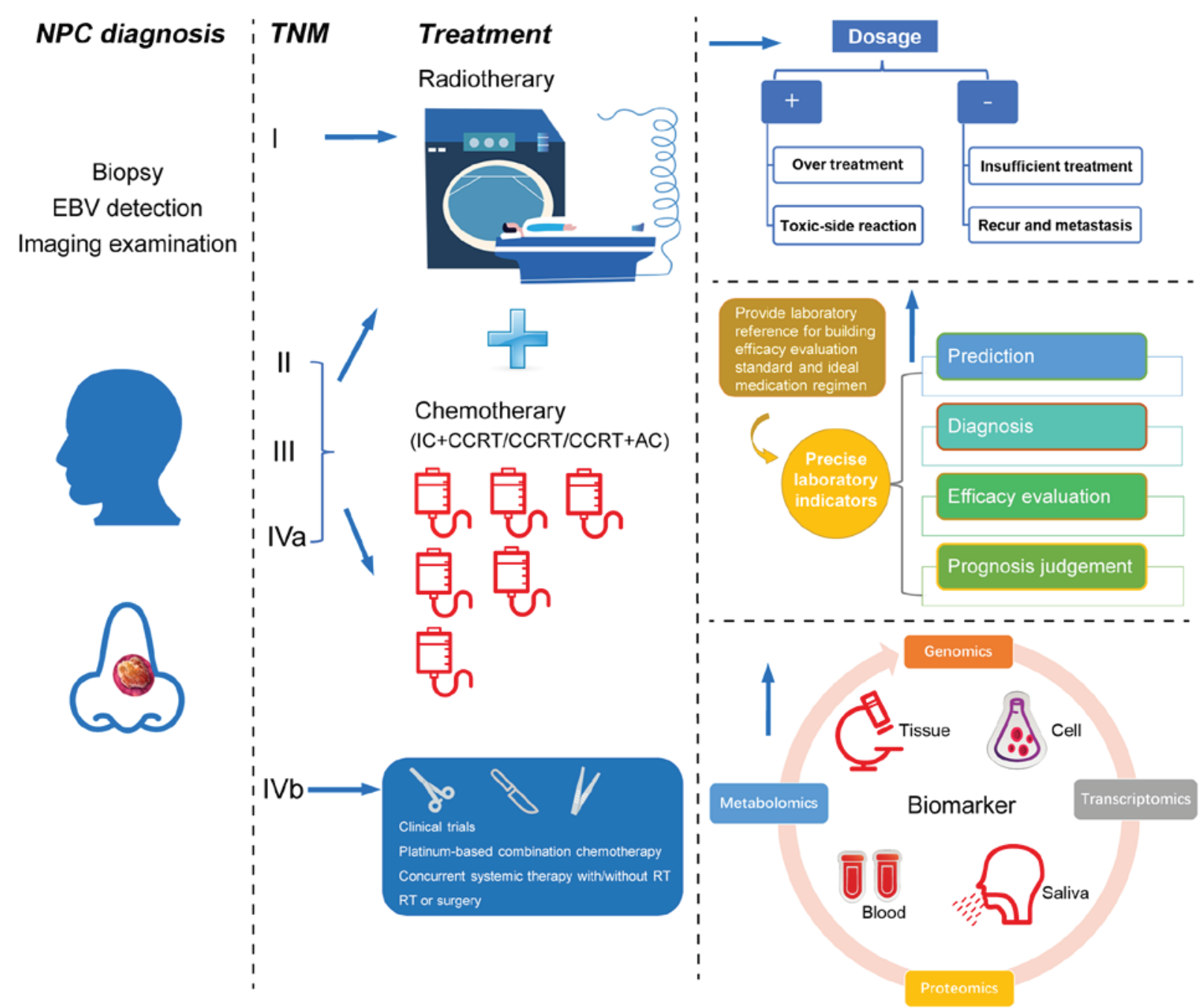

Figure 1. Role of biomarkers in NPC diagnosis, treatment, and prognosis. Biomarkers can effectively predict the risk of NPC incidence and reduce the number of patients with a locoregionally advanced stage through early diagnosis. For patients at different stages, biomarkers can effectively assist physicians to carry out clinical individualized treatment. This includes creating ideal regimens to improve the treatment effect and providing targets for the development of novel medication which avoid the toxic reactions caused by treatment. Effective biomarkers may also be used to accurately evaluate the prognosis of patients, as well as reduce the recurrence and distant metastasis of tumors. AC, adjuvant chemotherapy; CCRT, concurrent chemoradiotherapy; EBV, Epstein-Barr virus; IC, induction chemotherapy; NPC, nasopharyngeal carcinoma; TNM, tumor-node-metastasis.

DNA glycosylase 1 (hOGGl), integrin (ITG) $\alpha 2(36)$ and cyclophilin A26 (37) in patient cohorts from various regions, and confirmed the effect of loss-of-function mutations such as negative regulator NF- $\mathrm{KB}$ inhibitor $\alpha$ and cylindromatosis on NF- $\kappa B$ activity and NPC cell growth, respectively $(38,39)$. To date, no susceptibility genes have been approved for the early screening of NPC. The heterogeneity in the sample cohorts of different regions, races and pathological backgrounds is often highlighted as the main reason for the variation between the research results (7). Nevertheless, to the best of our knowledge, most studies did not verify the potential pathogenic genes. Some scholars think that because SNPs identified by GWAS are mostly minor alleles; therefore the main difficulty in further research, the large samples and failure to verify the downstream function of non-coding regions are the main reasons for the difficulty further research (33). In addition, the integration of multi-disciplinary research may provide a new method to interpret this complex problem, such as the joint effect of some specific regional environmental factors and traditional unhealthy diets on the incidence of NPC (40-42).
However, finding the interdisciplinary intersection point seems to be beyond the research objective itself.

In a whole exon sequencing study of 251 individuals from 97 polygenic families in Taiwan, Yu et al (43) found that 12 gene variations related to magnesium transport (Nuclear-interacting partner of anaplastic lymphoma kinase-like domain containing 1), EBV cell entry (ITG- $\beta 6$ ), modulation of EBV infection (bcl-2-like protein 12; and neural precursor cell expressed, developmentally down-regulated 4-like), telomere biology (cleft lip and palate transmembrane 1 like; bromodomain containing 2; and heterogeneous nuclear ribonucleoprotein $\mathrm{U}$ ), modulation of cAMP signaling (rap guanine nucleotide exchange factor 3), DNA repair (protein kinase, DNA-activated, catalytic subunit; and mutL homolog 1) and the Notch signaling pathway (notch receptor 1 ; and $\delta$-like canonical notch ligand 3), play important roles in activating $\mathrm{T}$ cells to respond to EBV infection. Liu et al (44) verified these mutations in a later study and emphasized the important role of telomere length maintenance in NPC etiology. Two other studies from the USA and Tunisia showed the potential of 
mixed-lineage leukemia 3; major histocompatibility complex, class I, A*26; major histocompatibility complex, class I, A*30; and major histocompatibility complex, class II, DR $\beta 1 * 10$ in screening high-risk NPC family members, respectively $(45,46)$. The study pointed out that the relative potential risk of NPC among family members who have first-degree relatives diagnosed with NPC is increased eight-fold (47). For some high-risk countries or regions, it is still necessary to strengthen the mining of familial NPC susceptibility genes for the development of related gene detection and early diagnosis.

Patients with NPC can develop various degrees of oral mucositis, xerostomia, myelosuppression and other toxic reactions while receiving intensity-modulated RT (IMRT) (48). Furthermore, cancer recurrence and distant metastasis can occur in 30-40\% of patients with NPC after treatment, indicating that the response of NPC to RT is subject to individual variation (49). Because tumor cells can repair radiation-induced damage in various ways, some genes related to radiosensitivity are often used to predict the therapeutic effect and prognosis of NPC (16,48-50). Ma et al (51) analyzed the genotypes of angiogenesis-related genes in 180 patients with NPC using Sequenom MassARRAY and found that EDN1-rs1800541, rs2071942 and rs5370 can be used as risk predictors of radiation-induced oral mucositis, xerostomia and myelosuppression, respectively. Le et al (52) screened SNPs of 24 patients with NPC by WGS and found that rs11081899-A, located in the 5'-untranslated region of the zinc finger protein 24 gene, is the genetic predisposing factor of radiation-induced oral mucositis. Yu et al (53) analyzed 9 potential functional SNPs in four genes in the Wnt/ $\beta$-catenin pathway and found that patients carrying catenin $\beta-1$ rs1880481, rs3864004; glycogen synthase kinase-3 $\beta$ rs 3755557 ; or adenomatous polyposis coli rs454886, may have a poor prognosis and can develop radiation-induced dermatitis and oral mucositis. Furthermore, several studies have discussed the potential of SNPs of different genes, such as X-ray repair cross-complementing 1 (XRCC1) rs25489, XRCC1 Codon399, valosin-containing protein rs2074549 and rcalcitonin receptor rs2528521 in the base excision repair pathway and the endoplasmic reticulum stress pathway to predict the therapeutic effect and toxic reactions in NPC (54-57). However, it is worth noting that the cases involved in these studies included a number of patients with stages III-IVa NPC. Although these patients also needed standard RT, the additional effect of chemotherapeutic drugs in CCRT on blood composition is an important factor that cannot be ignored. Tan et al (58) found that the vascular endothelial growth factor-460C allele had a significant association with NPC invasiveness of grades 2-3 cervical lymph node metastasis and compared with CCRT, in NPC with-460 T/C polymorphisms, patients may not benefit from IC+CCRT in terms of the OS, LRFS, DMFS and PFS, which again emphasizes the contradicted value of using IC for clinical application.

In addition to the inherent susceptibility genes of humans, EBV, a category 1 carcinogenic virus identified by IARC, is detectable in $100 \%$ of patients with undifferentiated non-keratinizing NPC in endemic areas (especially in East and Southeast Asian countries, such as China). The mechanisms of EBV infection, carcinogenesis, modification of epigenetic profiles, immune escape and maintenance of tumor cell survival are deeply understood and have been previously described (59). Therefore, based on this close relationship, EBV DNA or antibodies can be used as an early diagnostic tool to screen for patients with NPC. In recent years, the A157154C polymorphism of the A73 gene and the RPMS1 genotype of EBV have been identified for their susceptibility to NPC $(60,61)$. In particular, the combination of EBV-viral capsid antigen (VCA) IgA and EBV-early antigen IgA showed better sensitivity and specificity than EBV-DNA in screening NPC high-risk family individuals (62). However, a large cohort study showed that the sensitivity and specificity of plasma EBV-DNA for the diagnosis of early asymptomatic NPC were 97.1 and $98.6 \%$, respectively (63). The authors speculated that the difference in the results may be owing to variations of EBV DNA content in the serum or plasma, clinical stage and pathological classification of the patients (64). Furthermore, previous studies have found no significant difference in the exact load of viral nucleic acid between patients with NPC and non-cancerous controls, and an increase in EBV levels was noted in asymptomatic carriers and patients with mononucleosis and pharyngitis $(65,66)$. Banko et al $(67)$ found that P-Thr-sv-5 showed carcinoma-specific Epstein-Barr nuclear antigen 1 (EBNA1) variability in tissues and plasma of patients with undifferentiated NPC, monocytosis syndrome and renal transplantation by using nested PCR and considered that the identification of this subvariant should be used as a viral screening marker for the identification of NPC. However, further in vitro experiments are needed to confirm whether the additional amino acid substitution of this subvariant will affect the function of EBNA1 in regulating the replication and transcription of EBV genes and/or change the effect of EBNA1 on the transcription of other genes in host cells (68). In terms of efficacy evaluation and prognosis, Hui et al (69) detected SNPs of the excision repair 1 endonuclease non-catalytic subunit $(E R C C 1)$ gene in the plasma of patients with EBV-positive and EBV-negative NPC after RT in a clinical trial. The results showed that the ERCC1 C118T genotype was a powerful predictor for a good prognosis of patients with EBV-negative NPC, which could confirm the effect of RT and avoid serious toxic reactions caused by the additional adjuvant CT. Nevertheless, in view of some potential influencing factors mentioned in the aforementioned study, whether the objectivity of the results can be affected by different clinical stages, treatment methods and other confounding factors remains to be further explored.

\section{NPC biomarkers discovered through transcriptomics}

Transcriptomics studies gene transcription and its regulation at the RNA level (70). It explores variations in gene expression using sequencing technology and reveals the mechanism of specific regulatory genes in a pathological condition through cell phenotypes and functional research (71). In contrast to genomics, transcriptomics emphasizes the concept of time and space, which enables it not only to identify the phenotypic attribution of cells, but also to distinguish subtypes of diseases and to discriminate the various reactions caused by medications, as well as describe the survival rates of patients (72).

Previous studies have shown that micro RNAs (miRNAs/miRs) can play an important role in the occurrence, 
invasion, metastasis and immune escape of NPC, and their stable expression in the peripheral circulation can not only be used as reliable markers for the early diagnosis of NPC but may also help in the effective prediction of the therapeutic effect and prognosis (73-75). Therefore, studies into miRNAs have gained a leading position in the field of transcriptomics in recent years (73). Recently, several miRNAs with good diagnostic ability have been found using PCR or microarray technology (76-83). Two types of EBV-encoded miRNAs, miR-BamHI A rightward transcripts (BART)7-3p and miR-BART13-3p, showed a remarkable potential for the early diagnosis of NPC (76). It is worth noting that as the first virus-encoding miRNAs, 44 mature miRNAs encoded by EBV have been confirmed to play an important regulatory role in the carcinogenesis and progression of NPC. Although the function and clinical value of these miRNAs has been explored comprehensively, some of the identified biomarkers still lack functional verification $(84,85)$. In addition to the aforementioned problems, most of these studies lack functional analysis of the identified potential biomarkers. Because of the small molecular weight of miRNAs and their low content in the serum or plasma, it is difficult to fully reflect the expression abundance of these biomarkers. Therefore, the sensitivity and specificity of miRNAs, especially EBV miRNAs, were not satisfactory $(78,80)$. Furthermore, although a previous study confirmed the mechanism of action of miRNAs in various stages of NPC at the molecular level, more clinical verification is needed to assess the application of these potential biomarkers (73). Tumor-educated platelets, which are believed to be able to accurately diagnose for various types of cancer in liquid biopsies, have recently been used as early diagnostic biomarkers for NPC. Wang et al (81) detected the expression of miR-34c-3p and miR-18a-5p in the platelets of patients with NPC and healthy controls, and found a reasonable diagnostic potential of the two miRNAs with a sensitivity of $92.59 \%$, specificity of $86.11 \%$ and an area under the curve (AUC) value of 0.954 . Although additional validation and functional analysis were not conducted, Best et al (86) pointed out that platelets, as anucleated cell fragments in the blood circulation, can modulate the splicing of their pre-mRNAs in response to signals from cancer cells and then change their transcriptome and molecular signals. Compared with other samples, platelets do not contain nuclei and are less interfered with by genomic DNA; therefore, their RNA expression truly reflects the pathological progression of tumors, providing a more valuable new platform for biomarker research (81).

Compared to PCR, microarray-based high-throughput technology has many advantages. However, it is often not sufficient to use only common features, such as gene names, for comparisons across different microarray platforms $(7,8)$. The main reason for this is that different probes used in different studies may not have the tagging gene name or different probes of the same gene may not give similar signals (73). Therefore, it has been suggested that additional verification by PCR is necessary for the final identification of biomarkers in an independent verification cohort, as it allows the evaluation of gene expression more reliably (7). Wu et al (83) detected the miRNA expression profile in NPC saliva for the first time. They applied the stacking-hybridized universal tag (SHUT) miRNA array and quantitative PCR (qPCR) technology to compare the
miRNA expression levels in patients with NPC and healthy controls, and determined that the sensitivity and specificity of 12 differential miRNAs used to distinguish between patients with NPC and a healthy population were 100 and $96 \%$, respectively. Bioinformatics analysis showed that these differentially expressed miRNAs play an important role in the development of NPC by regulating their target genes, such as platelet-derived growth factor receptor $\alpha ; R A C 1$; inhibitor of NF- $\mathrm{NB}$ kinase regulatory subunit $\gamma$; X-linked inhibitor of apoptosis; and protein phosphatase, $\mathrm{Mg}^{2+} / \mathrm{Mn}^{2+}$ dependent 1D. Furthermore, Li et al (87) reported the ability of hsa-mir-1281 and hsa-mir-6732-3p to evaluate the efficacy of RT for NPC by assessing the serum of patients with different RT sensitivities. Unsatisfactory AUC values of both miRNAs were found $(0.750$ for hsa-mir-1281 and 0.696 for hsa-mir-6732-3p); however the small sample size included in the study, as well as the fact that $>50 \%$ of patients had stages III-IVa NPC and did not receive RT alone, may have affected the results.

Long non-coding (lnc)RNAs also play important roles at the transcriptomic level, performing a regulatory role in NPC epigenetics (88). Previous studies have confirmed that lncRNAs, miRNAs and EBV products can target each other and share common signaling pathways to form a complex molecular regulatory network (89). In recent years, novel lncRNAs have been identified through high-throughput sequencing to impact on the occurrence, progression, recurrence, metastasis and prognosis of NPC. However, the purpose of these studies was mainly to determine the mechanisms of action and treatment targets of NPC in different stages, rather than to identify biomarkers for NPC (88). He et al (90) used reverse transcription (RT)-qPCR to detect NPC-related lncRNAs in four different NPC cell lines and normal nasopharyngeal epithelial cells, as well as in the serum of patients with NPC, patients with chronic nasopharyngitis, EBV carriers and healthy controls. The AUC value of the three combined lncRNAs, including metastasis associated with lung adenocarcinoma transcript, actin filament-associated protein 1-antisense RNA1 and AL359062, to discriminate between patients with NPC and the healthy population, was 0.918 . Furthermore, they found that the expression levels of these three IncRNAs significantly decreased in serum after treatment, thus confirming their potential as early diagnostic and efficacy evaluation biomarkers for NPC. Based on the current research progress, there is still a huge space for researchers to explore the value of lncRNAs as NPC biomarkers through liquid biopsy. High-throughput based second-generation sequencing technology does not rely on the prior knowledge of genomic information, which helps to identify new splicing points and mutations. Similar to miRNAs, an increasing number of IncRNAs have been found to be significantly related to the mechanism of action behind the radiosensitivity of NPC, but there are few clinical studies involving noninvasive detection (91).

Recently, RNA-sequencing has been used to detect the transcriptional profile of peripheral blood mononuclear cells in patients with NPC before and after RT. Although a study has reported that the 11 genes that have been found can be used as biomarkers to evaluate the prognosis of NPC after RT, a very small sample size was used and they did not establish a model for verification (92). In another study, Shuai et al (93) detected 
increased expression levels of circular RNA_0000285 at the homeodomain interacting protein kinase 3 locus in NPC cells, tissues and serum samples, which could be used as a biomarker to predict the radiosensitivity of NPC. However, their study also lacked a diagnostic analysis of the sensitivity and specificity of biomarkers. It should be noted that the interaction test is a common statistical method to confirm the identified differential genes as potential biomarkers and this method is usually based on statistical models (94). Therefore, although several potential NPC biomarkers have been found in various studies, owing to the interference of multiple factors, there are still no widely verified and accepted biomarkers in the field of transcriptomics.

\section{NPC biomarkers discovered through proteomics}

Proteins are involved in many important physiological functions, such as immunity, coagulation, substance exchange, transportation, metabolism and signaling pathway regulation (95). Their composition and expression levels are often affected by the pathological state of the body (96). Proteomics is a research field that is based on the protein expression profile of the normal human body. Proteins with significant differences in expression levels can be identified by screening and comparing all of the proteins expressed in the cells, tissues or blood of individuals (97). With the assistance of continuous innovation of high-throughput technology, an increasing number of proteins related to the pathogenesis of diseases have been found, allowing for further elucidation of the pathogenesis behind various diseases and the identification of molecular biomarkers and novel drug targets (98).

Several studies have shown that gene mutations in cancer often cause abnormal expression levels of corresponding proteins and such expression can be dynamically changed following damage to the DNA of cancer cells following therapies $(7,9)$. Furthermore, tumor cells can capture many protein decomposition products from normal tissues to synthesize the proteins they need, resulting in significant changes in protein expression levels due to increased anabolism and catabolism (99). Therefore, the proteins involved in carcinogenesis are considered functional molecules that can reflect the real-time state of disease progression and are used in the study of NPC biomarkers (7). Previous studies have identified various protein markers in different NPC cell lines, tissues and bodily fluid samples, including EBV-encoded proteins such as latent membrane protein (LMP)1, LMP2A and EBV nuclear antigens $(9,25,99)$. Owing to the accumulated knowledge from these studies, the mechanism of protein action in each stage of NPC has been further clarified and multiple protein-protein interaction networks have been established based on various signaling pathways (100). This has laid a foundation for future research, based on the phosphorylation, ubiquitination, sulfation, methylation and sumoylation of NPC protein markers (101).

Compared with 2D fluorescence difference gel electrophoresis, isobaric tags for relative and absolute quantification; matrix-assisted laser desorption/ionization time-of-flight mass spectrometry; surface-enhanced laser desorption/ionization time of flight mass spectrometry; and other technologies used in earlier studies, proteomics has made significant progress in the high-throughput technology of relative and absolute quantitation in recent years. For example, data-independent acquisition (used for comparative proteomics studies), parallel reaction monitoring (PRM) and phosphorylated PRM (used to study targeted proteomics), have improved significantly in the integrity of scanning, reproducibility, stability of results and the quantitative ability, and have been applied to study tumor biomarkers $(7,9,25,102-105)$. However, studies on NPC proteomics based on bodily fluid samples have not employed these new technologies and have shown a decreasing trend in the study number compared with earlier studies (106-111). The reasons for this are not clear, but it may be related to the difficulty in obtaining samples, the equipment conditions of the laboratory and the cost of testing.

Recently, Sun et al (106) used RT-qPCR to detect the mRNA expression levels of insulin receptor substrate 1 (IRS-1) in 133 patients with NPC and 104 healthy controls. The sensitivity and specificity of IRS-1 in the early diagnosis of NPC were 88.0 and 77.9\%, respectively. Coghill et al (107) applied a protein microarray to detect the anti-EBV-IgG and IgA antibody responses in 607 residents of Taiwan and found significant differences in the anti-EBV antibody levels of 60-IgA and 73-IgG between patients with NPC and healthy controls. The sensitivity and specificity of EBV VCA-IgA to distinguish patients with NPC were 66.7 and $95.0 \%$, respectively, with an AUC value of 0.811. In another study, 2D gel electrophoresis, ultra-performance liquid chromatography-tandem mass spectrometry (UPLC-MS/MS) and ELISAs were used to detect the serum auto-antibody levels of peroxiredoxin (PRDX)2 and PRDX3 in patients with NPC and CNE2 cells. Levels of PRDX2 and PRDX3 were significantly higher in patients with NPC and CNE2 cells than in the normal controls (108). In addition, Gong et al (109) detected cytokines in the serum of various cancer patients using antibody array technology. ELISA validation results showed that nine cytokines could be used as potential biomarkers for differential diagnosis and prognosis of NPC.

In terms of efficacy evaluation and prognosis, like genomics and transcriptomics, the evaluation of NPC radiosensitivity is still the main research focus of proteomics $(17,110)$. NPC cells develop resistance to RT, which involves cell cycle regulation, apoptosis and anti-apoptosis, as well as DNA damage and repair, which may lead to the abnormal protein synthesis of NPC cells and affect the protein expression level in blood. This difference can be found by assessing the serum protein expression levels of RT-sensitive patients $(7,9)$. Based on this hypothesis, Zhang et al (17) used the tandem mass tag method coupled with UPLC-MS/MS to detect the serum protein profile in patients with NPC with different RT effects. They found that the sensitivity and specificity of the combined five identified differential proteins, such as secreted protein acidic and rich in cysteine, serpin family $\mathrm{D}$ member 1S, complement C4B, peptidylprolyl lsomerase B and family with sequence similarity 173 member A to distinguish patients with RT resistance, were 94.1 and $92.6 \%$, respectively, and the AUC value was 0.968 . In addition, since there is currently no effective predictor for NPC recurrence, Meng et al (110) used the same technique to compare the serum protein expression levels in patients with NPC who developed recurrence. The results of ELISA validation revealed that differentially 
expressed calmodulin can be used as a potential biomarker for the diagnosis of NPC recurrence.

Although some researchers are still using bodily fluid samples to promote the research of NPC proteomics, the following obstacles need to be overcome to transform proteomics biomarkers into clinical applications. Initially, as mentioned above, the high-throughput technologies used in the existing studies are still relatively old. The limitations of these technologies in protein quantification, data collection, low abundance protein detection and repeatability restrict the discovery of some novel biomarkers with clinical significance $(98,111)$. Moreover, most studies are not adequate with respect to the study design, which could affect the results by a variety of confounding factors, thus interfering with the sensitivity and specificity of the markers (94). For example, in the study of efficacy evaluation and prognostic biomarkers, researchers should focus on the dual effects of RT and CT on blood components, as well as the use of recognized and unified clinical efficacy evaluation standards and external data sets to fully evaluate and verify the results (112). Eventually, there is a significant difference in the application of the detection technology, pathological background of samples and statistical analysis tools, and the functional research often cannot be combined with clinical studies $(113,114)$. These factors are bound to affect the repeated validation of identified biomarkers in different laboratories. In 2016, the US cancer moonshot 2020 officially launched a plan for precision medicine (115). Through the establishment of a data system for characterization of gene information and protein information, using genomics and proteomics as routine detection methods may provide more accurate guidance for individualized cancer treatment in the future. Therefore, it is believed that in the coming years, there will be an upsurge in NPC proteomics research based on high-throughput technology (116).

\section{NPC biomarkers discovered through metabolomics}

Briefly, metabolomics is a subject that conducts both qualitative and quantitative analysis of all low molecular weight metabolites $(<1,000)$ of a certain organism or cell at the same time in a specific physiological period (117). It is based on high-throughput mass spectrometry technology, cluster index analysis and data processing, combined with information modeling and system integration, to screen and identify the differential metabolites correlated to the disease phenotype (118). Compared with other omics fields, metabolomics is rather new and has currently attracted substantial interest in the field of tumor biomarkers (119).

As downstream products of genes and proteins, bioactive metabolites are an important component of systems biology, because they can directly regulate biological processes and phenotypes by regulating the main mechanisms of action behind the functions of DNA, RNA and protein (117). Metabolic change is an important feature of cancer. To maintain continuous proliferation and survival after treatment, cancer cells must adjust their metabolism and nutritional needs (120). Metabolic disorders in cancer cells can further affect the expression of cell surface markers through a variety of functional signaling pathways (121). The study of metabolic phenotypes can help to find more reliable evidence for the regulatory mechanism of the system that occurs or has occurred, rather than predicting the possible or upcoming changes. Therefore, metabolomics studies are not limited to the discovery of specific biomarkers. The exploration of characteristic pathological pathways and therapeutic targets can provide a more accurate direction for the development of novel medications $(122,123)$.

Previously, a gas chromatography-mass spectrometry (GC-MS)-based metabolomics platform was the main technology employed to study bodily fluid samples. Tang et al (124) used this technology to detect 51 serum metabolites in 49 patients with NPC, 37 with laryngeal cancer and 40 healthy controls. Through the validation of differential metabolites in tissues and in the serum of patients with NPC, they found that kynurenine, $\mathrm{N}$-acetylglucosaminylamine, $\mathrm{N}$-acetylglucosamine and hydroxyphenylpyruvate can be used as potential biomarkers for the early diagnosis of NPC. Furthermore, they observed changes in these four metabolites at three time periods after RT and found that the high expression was closely related to cancer recurrence and distant metastasis, thereby confirming the efficacy and prognosis of these potential diagnostic markers. In another study, Yi et al (125) compared the serum metabolic profile in 100 patients with NPC to that in healthy controls and found that the sensitivity and specificity of seven metabolites (glucose, linoleic acid, stearic acid, arachidonic acid, proline, $\beta$-hydroxy butyrate and glycerol 1-hexadecanoate) for NPC diagnosis were 88.0 and $92.0 \%$, respectively. Furthermore, they found that $\beta$-hydroxy butyrate and arachidonic acid can be used as evaluation indexes for the favorable prognosis of RT.

Along with the deepening understanding of NPC, the TNM staging system, RECIST and NCCN guidelines have changed significantly $(12,23)$. Compared with early two-dimensional RT, the application of IMRT technology has significantly improved the treatment of NPC (126). Therefore, there is no need to evaluate the aforementioned studies in these aspects. However, it is important to ensure early studies have carried out long-term follow-up of patients, which may help to further verify the dynamic performance of the biomarkers. Compared with GC-MS, high-throughput, widely targeted metabolomics technology can solve the problem that non-targeted metabolomics methods cannot detect metabolites in batches, and may show higher sensitivity and high throughput performance in the known qualitative and quantitative analysis of metabolites, as well as in the detection of low-abundance metabolites. Therefore, it has been successfully applied to screen tumor biomarkers with the advantages of a self-built database to identify new metabolites $(127,128)$. However, in recent years, to the best of our knowledge, there have not been any reports on the identification of NPC biomarkers in this field. This may also indicate the research potential and value of this field.

\section{Key points for study design}

Obtaining ideal biomarkers requires rigorous scientific study design and any design flaw will directly affect the experimental results and its clinical translational potential (94). Based on the different study purposes, the selection of case-cohort, bodily fluid samples and detection technology should be different, 
and the influence of tumor stages, treatment methods and experimental conditions should also be considered (129).

In the selection of case-cohort, the study of early diagnostic markers should try to avoid the inclusion of patients with locally advanced or distant metastasis, because the secretion of metastatic tumors in cervical lymph nodes or distant organs cannot be ignored in biomarker screening $(62,67,76,79-83$, $106,107,109)$. Although the smaller number of patients with early-stage disease is the biggest obstacle to conducting such studies, their blood composition may provide feedback on the simplest specific marker information (5). Similarly, studies on biomarkers for NPC radiosensitivity should also focus on early-stage patients as most patients in stages III-IVa need additional CT $(17,51,87)$. A previous study showed that the addition of cisplatin and paclitaxel can affect the expression levels of malondialdehyde, superoxide dismutase, catenin, glutathione, r-glutamyl cysteingyl and glycine in the blood of patients with NPC (130). Another study also confirmed that CCRT, RT or IC treatment may lead to varied changes in the distribution of metabolites in the serum of patients with head and neck cancer (131). Conversely, in a phase III clinical trial, Sun et al (112) found that the total IC efficacy of the combination of docetaxel, cisplatin and fluorouracil in the treatment of stages III and IV head and neck squamous cell carcinoma was significantly higher than that of the combination of cisplatin and fluorouracil (76.3 vs. 52.9\%). However, this difference disappeared after CCRT (75 vs. $73.9 \%$ ), indicating that RT may also have a significant effect on the efficacy of IC.

Serum and plasma are the most commonly studied bodily fluids (132). Several studies have found that compared with the coagulation of serum during collection, the miRNA-aligned reads produced in rat plasma during detection were twice as high as those produced by serum, but this difference was not found in human blood (133). However, among the 64 blood test indexes with statistical differences introduced by the WHO, 56 showed that the detection stability of plasma was significantly better than that of serum (134). Although this observation has been put into practice in several studies on the relationship between NPC and EBV, further verification and discussions are required to obtain general recognition $(77,135)$. In addition, the collection, processing and storage of samples will have a significant effect on the experimental results (93). Taking the use of anticoagulants as an example, the protocol of sample handling and storage in the British biobank has clearly defined the scope of application of anticoagulants such as citrate, EDTA and heparin (136). Several other reports have also discussed the effects of various storage and processing methods on the composition of the blood $(137,138)$. Thus, it can be seen that the normalization of procedures will help standardize the handling of samples in future studies and improve the quality of molecular detection.

For the detection technology, there is no doubt that new technology can often make up for the shortcomings of previous ones and that they are conducive to obtaining more specific biomarkers with respect to screening range and convenience of data analysis (139). Through a review of biomarker studies on the bodily fluid samples of patients with NPC in recent years, it is clear that there is a lack of application of new technologies in current studies, especially in the field of proteomics and metabolomics. Because of funding constraints, researchers must often make a difficult choice between technology and sample size, which is the main reason most studies have difficulty in achieving authoritative recognition (140). In addition, although the screening and identification of biomarkers using bodily fluid samples have represented a complete story, it is still necessary to conduct cellular-based and molecular biology techniques to confirm the functions of these potential biomarkers.

\section{Conclusion and future perspectives}

Compared to the study of other common types of cancer, that of NPC is concentrated in Asian countries, specifically China, owing to the geographical distribution of its incidence $(1,3,10)$. Great progress has been made in clinical trials of this disease and the NCCN guidelines have been rewritten (12). Conversely, biomarker research based on liquid biopsies is lagging, especially in the fields of proteomics and metabolomics, which limits the advancement of relevant research to a certain extent (7-9,13-15,17,100,124,125). For example, IC, as a standard initial method for systemic treatment of LA-NPC, the results of clinical trials show that not all patients can benefit from its use (15). Compared with CCRT alone, the prognosis of a considerable number of LA-NPC patients has not been significantly improved due to the addition of IC, indicating that they have suffered unnecessary over treatment (22). Conversely, in those who have extended the survival by IC+CCRT, 20-30\% patients with LA-NPC still present tumor relapse and metastasis, suggesting that the current clinical application of IC may also provide insufficient treatment $(13,14)$. Therefore, it is urgent to identify molecular biomarkers to predict the short-term efficacy of IC. In addition, because of serious toxic reactions in the blood, digestive tract, skin, nervous system, liver and kidney caused by RT and CT, new therapeutic targets are necessary for the development of new, safer medications $(10,20,21)$.Therefore, future research on NPC biomarkers should gradually extend to the evaluation of efficacy and prognosis based on early diagnosis to provide sufficient laboratory data for the clinical research of NPC.

With the development of tumor biomarkers, it is impossible to fully explain the complex biological processes and network regulation behind the carcinogenesis and progression of tumors from a systematic perspective only by studying a single omics (141). Therefore, multi-omics integration has become a new trend to promote the research and development of tumor biomarkers (142). The pathogenesis behind the changes required for cancer, gene mutation, transcriptional regulation, protein synthesis and metabolic changes constitute a systematic mechanism (143). Based on these relationships, the integration of two or more kinds of omics research and the use of machine learning methods to carry out association analysis on molecules at multiple levels, could make up for the lack of data caused by single omics analysis and reduce the probability of false positive results. This would allow researchers to study the phenotype and regulatory mechanisms of action in biological models more effectively and investigate complex scientific problems more comprehensively. By using this strategy, NPC biomarkers may effectively compensate for the deficiencies in early omics research, promoting the overall development of NPC clinical research. 
Collectively, novel clinical issues, scientific study design, cutting-edge high-throughput technology, integrated multi-omics platforms, extensive screening of large-scale cohort, systematic functional analysis, ensemble learning and comprehensive and in-depth validation are inevitable methods that are required to enhance research into NPC biomarkers using bodily fluid samples.

\section{Acknowledgements}

Not applicable.

\section{Funding}

The present study was supported by the Shaoguan Science and Technology Plan Projects in 2020 (grant no. 200812094530421).

\section{Availability of data and materials}

Not applicable.

\section{Authors' contributions}

JCL and SQZ conceived and designed the review. SQZ, SXL and YSH consulted the literature. SQZ analyzed the literature and drafted the manuscript. YSH produced the figure. JCL, SMP and HBC critically revised the article for important intellectual content and assisted in the literature search for this review article. All authors read and approved the final version of this manuscript.

\section{Ethics approval and consent to participate}

Not applicable.

\section{Patient consent for publication}

Not applicable.

\section{Competing interests}

All the authors declare that they have no competing interests.

\section{References}

1. Chen YP, Chan ATC, Le QT, Blanchard P, Sun Y and Ma J: Nasopharyngeal carcinoma. Lancet 394: 64-80, 2019.

2. Bray F, Ferlay J, Soerjomataram I, Siegel RL, Torre LA and Jemal A: Global cancer statistics 2018: GLOBOCAN estimates of incidence and mortality worldwide for 36 cancers in 185 countries. CA Cancer J Clin 68: 394-424, 2018.

3. Wee JT, Ha TC, Loong SL and Qian CN: Is nasopharyngeal cancer really a 'Cantonese cancer'? Chin J Cancer 29: 517-526, 2010.

4. Tang LL, Chen YP, Mao YP, Wang ZX, Guo R, Chen L, Tian L, Lin AH, Li L, Sun Y and Ma J: Validation of the 8th edition of the uicc/ajcc staging system for nasopharyngeal carcinoma from endemic areas in the intensity-modulated radiotherapy era. J Natl Compr Canc Netw 15: 913-919, 2017.

5. Roy Chattopadhyay N, Das P, Chatterjee K and Choudhuri T: Higher incidence of nasopharyngeal carcinoma in some regions in the world confers for interplay between genetic factors and external stimuli. Drug Discov Ther 11: 170-180, 2017.

6. Chang ET and Adami HO: The enigmatic epidemiology of nasopharyngeal carcinoma. Cancer Epidemiol Biomarkers Prev 15: 1765-1777, 2006.
7. Janvilisri T: Omics-based identification of biomarkers for nasopharyngeal carcinoma. Dis Markers 2015: 762128, 2015.

8. Lee KT, Tan JK, Lam AK and Gan SY: MicroRNAs serving as potential biomarkers and therapeutic targets in nasopharyngeal carcinoma: A critical review. Crit Rev Oncol Hematol 103: 1-9, 2016.

9. Xiao L, Xiao T, Wang ZM, Cho WC and Xiao ZQ: Biomarker discovery of nasopharyngeal carcinoma by proteomics. Expert Rev Proteomics 11: 215-225, 2014

10. Zhang Y, Chen L, Hu GQ, Zhang N, Zhu XD, Yang KY, Jin F, Shi M, Chen YP, Hu WH, et al: Gemcitabine and cisplatin induction chemotherapy in nasopharyngeal carcinoma. N Engl J Med 381: 1124-1135, 2019.

11. Song C, Cheng P, Cheng J, Zhang Y, Sun M, Xie S and Zhang X: Differential diagnosis of nasopharyngeal carcinoma and nasopharyngeal lymphoma based on DCE-MRI and RESOLVE-DWI. Eur Radiol 30: 110-118, 2020.

12. Colevas AD, Yom SS, Pfister DG, Spencer S, Adelstein D, Adkins D, Brizel DM, Burtness B, Busse PM, Caudell JJ, et al: NCCN guidelines insights: Head and neck cancers, version 1.2018. J Natl Compr Canc Netw 16: 479-490, 2018.

13. Sun XS, Xiao BB, Lu ZJ, Liu SL, Chen QY, Yuan L, Tang LQ and Mai HQ: Stratification of candidates for induction chemotherapy in Stage III-IV nasopharyngeal carcinoma: A large cohort study based on a comprehensive prognostic model. Front Oncol 28: $255,2020$.

14. Wang YW, Ho SY, Lee SW, Chen CC, Litsu S, Huang WT, Yang CC, Lin CH, Chen HY and Lin LC: Induction chemotherapy improved long term outcomes in stage IV locoregional advanced nasopharyngeal carcinoma. Int J Med Sci 17: 568-576, 2020.

15. Cao SM, Yang Q, Guo L, Mai HQ, Mo HY, Cao KJ, Qian CN, Zhao C, Xiang YQ, Zhang XP, et al: Neoadjuvant chemotherapy followed by concurrent chemoradiotherapy versus concurrent chemoradiotherapy alone in locoregionally advanced nasopharyngeal carcinoma: A phase III multicentre randomised controlled trial. Eur J Cancer 75: 14-23, 2017.

16. Jin BY, Zhang GY, Lin KR, Chen XP, Cui JH, Wang YJ and Luo W: Changes of plasma cytokines and chemokines expression level in nasopharyngeal carcinoma patients after treatment with definitive intensity-modulated radiotherapy (IMRT). PLoS One 12: $\mathrm{e} 0172264,2017$.

17. Zhang G, Zhang K, Li C, Li Y, Li Z, Li N, Zhou Q and Shen L: Serum proteomics identify potential biomarkers for nasopharyngeal carcinoma sensitivity to radiotherapy. Biosci Rep: May 14 2019 (Epub ahead of print). doi: 10.1042/BSR20190027.

18. Aftab O, Liao S, Zhang R, Tang N,Luo M,Zhang B, Shahi S, Rai R, Ali J and Jiang W: Efficacy and safety of intensity-modulated radiotherapy alone versus intensity-modulated radiotherapy plus chemotherapy for treatment of intermediate-risk nasopharyngeal carcinoma. Radiat Oncol 15: 66, 2020.

19. Wang HY, Chang YL, To KF, Hwang JS, Mai HQ, Feng YF, Chang ET, Wang CP, Kam MK, Cheah SL, et al: A new prognostic histopathologic classification of nasopharyngeal carcinoma. Chin J Cancer 35: 41, 2016.

20. Al-Sarraf M, LeBlanc M, Giri PG, Fu KK, Cooper J, Vuong T, Forastiere AA, Adams G, Sakr WA, Schuller DE and Ensley JF: Chemoradiotherapy versus radiotherapy in patients with advanced nasopharyngeal cancer: Phase III randomized intergroup study 0099. J Clin Oncol 16:1310-1317, 1998.

21. Lee AW, Tung SY, Ngan RK, Chappell R, Chua DT, Liu TX, Siu L, Tan T, Chan LK, Ng WT, et al: Factors contributing to the efficacy of concurrent-adjuvant chemotherapy for locoregionally advanced nasopharyngeal carcinoma: Combined analysesof NPC-9901 and NPC-9902 trials. Eur J Cancer 47: 656-666, 2011.

22. Chen Y, Liu MZ, Liang SB, Zong JF, Mao YP, Tang LL, Guo Y, Lin AH, Zeng XF and Ma J: Preliminary results of a prospective randomized trial comparing concurrent chemoradiotherapy plus adjuvant chemotherapy with radiotherapy alone in patients with locoregionally advanced nasopharyngeal carcinoma in endemic regions of China. Int J Radiat Oncol Biol Phys 71: 1356-1364, 2008.

23. Liang H, Xu Y, Chen M, Zhong W, Wang M and Zhao J: Patterns of response in metastatic NSCLC during PD-1 or PD-L1 inhibitor therapy: Comparison of the RECIST 1.1 and iRECIST criteria. Thorac Cancer 11: 1068-1075, 2020.

24. Jiang W, Liu N, Chen XZ, Sun Y, Li B, Ren XY, Qin WF, Jiang N, $\mathrm{Xu}$ YF, Li YQ, et al: Genome-wide identification of a methylation gene panel as a prognostic biomarker in nasopharyngeal carcinoma. Mol Cancer Ther 14: 2864-2873, 2015. 
25. Chen ZT, Liang ZG and Zhu XD: A review: Proteomics in nasopharyngeal carcinoma. Int J Mol Sci 16: 15497-15530, 2015.

26. Del Giacco and Cattaneo C: Introduction to genomics. Methods Mol Biol 823: 79-88, 2012.

27. Berger MF and Mardis ER: The emerging clinical relevance of genomics in cancer medicine. Nat Rev Clin Oncol 15: 353-365, 2018

28. Donnelly D III, Aung PP and Jour G: The '-OMICS' facet of melanoma: Heterogeneity of genomic, proteomic and metabolomic biomarkers. Semin Cancer Biol 59: 165-174, 2019.

29. Tsang CM, Lui VW, Bruce JP, Pugh TJ and Lo KW: Translational genomics of nasopharyngeal cancer. Semin Cancer Biol 61: 84-100, 2020.

30. Yang H, Yu K, Zhang R, Li J, Wei X, Zhang Y, Zhang C, Xiao F, Zhao D, Lin X, et al: The HLA-DRB1 allele polymorphisms and nasopharyngeal carcinoma. Tumour Biol 37: 7119-7128, 2016.

31. Guo XG and Xia Y: The interleukin-18 promoter-607C $>$ A polymorphism contributes to nasopharyngeal carcinoma risk: Evidence from a meta-analysis including 1,886 subjects. Asian Pac J Cancer Prev 14: 7577-7781, 2013.

32. Yi M, Cai J, Li J, Chen S, Zeng Z, Peng Q, Ban Y, Zhou Y, Li X, Xiong W, et al: Rediscovery of $N F-\kappa B$ signaling in nasopharyngeal carcinoma: How genetic defects of NF- $\mathrm{BB}$ pathway interplay with EBV in driving oncogenesis? J Cell Physiol 233: 5537-5549, 2018

33. Tam V, Patel N, Turcotte M, Bosse Y, Paré G and Meyre D: Benefits and limitations of genome-wide association studies. Nat Rev Genet 20: 467-484, 2019.

34. Zhou P, Liu S, Ji NN, Zhang S, Wang P, Lin B, Yang P, Lin XT, Cai YZ, Wang ZM, et al: Association between variant alleles of major histocompatibility complex class II regulatory genes and nasopharyngeal carcinoma susceptibility. Eur J Cancer Prev 29:531-537, 2020.

35. Niu Y, Zhou G, Wang Y, Qin J, Ping J, Zhang Q, Han BW, Liu YX, Yang C, Zhai Y, et al: Association of MCP-1 promoter polymorphism with susceptibility to nasopharyngeal carcinoma. J Cell Biochem 120: 6661-6670, 2019.

36. Ban EZ, Lye MS, Chong PP, Yap YY, Lim SY and Abdul Rahman H: Haplotype CGC from XPD, hOGG1 and ITGA2 polymorphisms increases the risk of nasopharyngeal carcinoma in Malaysia. PLoS One 12: e0187200, 2017.

37. Lourembam DS, Singh AR, Sharma TD, Singh TS, Singh TR and Singh LS: Evaluation of risk factors for nasopharyngeal carcinoma in a high-risk area of India, the Northeastern region. Asian Pac J Cancer Prev 16: 4927-4935, 2015.

38. Zheng H, Dai W, Cheung AK, Ko JM, Kan R, Wong BW, Leong MM, Deng M, Kwok TC, Chan JY, et al: Whole-exome sequencing identifies multiple loss-of-function mutations of $\mathrm{NF}-\kappa \mathrm{B}$ pathway regulators in nasopharyngeal carcinoma. Proc Natl Acad Sci USA 113: 11283-11288, 2016.

39. Li YY, Chung GT, Lui VW, To KF, Ma BB, Chow C, Woo JK, Yip KY, Seo J, Hui EP, et al: Exome and genome sequencing of nasopharynx cancer identifies NF- $\kappa \mathrm{B}$ pathway activating mutations. Nature Commun 8: 14121, 2017.

40. Tsao SW, Yip YL, Ysang CM, Pang PS, Lau VM, Zhang G and Lo KW: Etiology factors of nasopharyngeal carcinoma. Oral Oncol 50: 330-338, 2014

41. Anderson EN Jr, Anderson ML and Ho HC: Environmental backgrounds of young Chinese nasopharyngeal carcinoma patients. IARC Sci Publ: 231-239, 1978.

42. Tan C, Chen H, Wu T and Xia C: The prediction of nasopharyngeal carcinoma mortality based on soil element levels in China. Biol Trace Elem Res 138: 139-147, 2010.

43. Yu G, Hsu WL, Coghill AE, Yu KJ, Wang CP, Lou PJ, Liu Z, Jones K, Vogt K, Wang M, et al: Whole-exome sequencing of nasopharyngeal carcinoma families reveals novel variants potentially involved in nasopharyngeal carcinoma. Sci Rep 9: 9916, 2019.

44. Liu Z, Goldstein AM, Hsu WL, Yu KJ, Chien YC, Ko JY, Jian JJ, Tsou YA, Leu YS, Liao LJ, et al: Evaluation of rare and common variants from suspected familial or sporadic nasopharyngeal carcinoma (NPC) susceptibility genes in sporadic NPC. Cancer Epidemiol Biomarkers Prev 28: 1682-1686, 2019.

45. Sasaki MM, Skol AD, Bao R, Rhodes LV, Chambers R, Vokes EE, Cohen EE and Onel K: Integrated genomic analysis suggests MLL3 is a novel candidate susceptibility gene for familial nasopharyngea carcinoma. Cancer Epidemiol Biomarkers Prev 24: 1222-1228, 2015.

46. Mokni-Baizing N, Gorgi Y, Elghourabi M, Makhlouf M, Boussen H, Gritli S, Elmay M, Gamoudi A and Elmay A HLA-A*26-A*30 and HLA-DRB1*10 could be predictors of nasopharyngeal carcinoma risk in high-risk Tunisian families. J Oral Sci 59: 289-296, 2017
47. Friborg J, Wohlfahrt J, Koch A, Storm H, Olsen OR and Melbye M: Cancer susceptibility in nasopharyngeal carcinoma families-a population-based cohort study. Cancer Res 65: 8567-8572, 2005

48. Kerns SL, West CM, Andreassen CN, Barnett GC, Bentzen SM, Burnet NG, Dekker A, De Ruysscher D, Dunning A, Parliament M, et al: Radiogenomics: The search for genetic predictors of radiotherapy response. Future Oncol 10: 2391-2406, 2014.

49. Chen $\mathrm{W}$ and $\mathrm{Hu} \mathrm{GH}$ : Biomarkers for enhancing the radiosensitivity of nasopharyngeal carcinoma. Cancer Biol Med 12: 23-32, 2015.

50. Rattay $\mathrm{T}$ and Talbot CJ: Finding the genetic determinants of adverse reactions to radiotherapy. Clin Oncol (R Coll Radiol) 26: 301-308, 2014.

51. Ma WL, Liu R, Huang LH, Zou C, Huang J, Wang J, Chen SJ, Meng XG, Yang JK, Li H, et al: Impact of polymorphisms in angiogenesis-related genes on clinical outcomes of radiotherapy in patients with nasopharyngeal carcinoma. Clin Exp Pharmacol Physiol 44: 539-548, 2017.

52. Le Z, Niu X, Chen Y, Ou X, Zhao G, Liu Q, Tu W, Hu C, Kong L and Liu Y: Predictive single nucleotide polymorphism markers for acute oral mucositis in patients with nasopharyngeal carcinoma treated with radiotherapy. Oncotarget 8: 63026-63037, 2017.

53. Yu J, Huang Y, Liu L, Wang J, Yin J, Huang L, Chen S, Li J, Yuan H, Yang G, et al: Genetic polymorphisms of Wnt $/ \beta$-catenin pathway genes are associated with the efficacy and toxicities of radiotherapy in patients with nasopharyngeal carcinoma. Oncotarget 7: 82528-82537, 2016.

54. Wang J, Guo C, Gong X, Ao F, Huang Y, Huang L, Tang Y, Jiang C, Xie X, Dong Q, et al: The impacts of genetic polymorphisms in genes of base excision repair pathway on the efficacy and acute toxicities of (chemo) radiotherapy in patients with nasopharyngeal carcinoma. Oncotarget 8: 78633-78641, 2017.

55. Zhai XM, Hu QC, Gu K, Wang JP, Zhang JN and Wu YW: Significance of XRCC1 Codon399 polymorphisms in Chinese patients with locally advanced nasopharyngeal carcinoma treated with radiation therapy. Asia Pac J Clin Oncol 12: e125-e132, 2016.

56. Chen H, Wu M, Li G, Hua L, Chen S and Huang H: Association between XRCC1 single-nucleotide polymorphism and acute radiation reaction in patients with nasopharyngeal carcinoma: A cohort study. Medicine (Baltimore) 96: e8202, 2017.

57. Guo XB, Ma WL, Liu LJ, Huang YL, Wang J, Huang LH, Peng XD, Yin JY, Li JG, Chen SJ, et al: Effects of gene polymorphisms in the endoplasmic reticulum stress pathway on clinical outcomes of chemoradiotherapy in Chinese patients with nasopharyngeal carcinoma. Acta Pharmacol Sin 38: 571-580, 2017.

58. Tan J, Jiang L, Cheng X, Wang C, Chen J, Huang X, Xie P, Xia D, Wang R and Zhang Y: Association between VEGF-460T/C gene polymorphism and clinical outcomes of nasopharyngeal carcinoma treated with intensity-modulated radiation therapy. Onco Targets Ther 10: 909-918, 2017.

59. Tsao SW, Tsang CM and Lo KW: Epstein-barr virus infection and nasopharyngeal carcinoma. Philos Trans R Soc Lond B Biol Sci 372: 20160270, 2017.

60. Shen JJ, Niu WN, Zhou M, Zhou F, Zhang HY and Wang L: Association of Epstein Barr virus A73 gene polymorphism with nasopharyngeal carcinoma. Genet Test Mol Biomarkers 19: $187-190,2015$.

61. Wu S, Liu W, Li H, Zhao Z, Yang Y, Xiao H, Song Y and Luo B: Conservation and polymorphism of EBV RPMS1 gene in EBV-associated tumors and healthy individuals from endemic and non-endemic nasopharyngeal carcinoma areas in China. Virus Res 250: 75-80, 2018

62. Tay JK, Chan SH, Lim CM, Siow CH, Goh HL and Loh KS: The role of Epstein-Barr virus DNA load and serology as screening tools for nasopharyngeal carcinoma. Otolaryngol Head Neck Surg 155: 274-280, 2016.

63. Chan KC, Woo JK, King A, Zee BC, Lam WK, Chan SL, Chu SW, Mak C, Tse IO, Leung SY, et al: Analysis of plasma Epstein-Barr virus DNA to screen for nasopharyngeal cancer. N Engl J Med 377:513-522, 2017.

64. Lam WK, Chan KC and Lo YM: Plasma Epstein-Barr virus DNA as an archetypal circulating tumour DNA marker. J Pathol 247: 641-649, 2019.

65. Zeng Z, Fan S, Zhang X, Li S, Zhou M, Xiong W, Tan M, Zhang W and Li G: Epstein-Barr virus-encoded small RNA 1 (EBER-1) could predict good prognosis in nasopharyngeal carcinoma. Clin Transl Oncol 18: 206-211, 2016. 
66. Arai A, Yamaguchi T, Komatsu H, Imadome K, Kurata M, Nagata $\mathrm{K}$ and Miura O: Infectious mononucleosis accompanied by clonal proliferation of EBV-infected cells and infection of CD8-positive cells. Int J Hematol 99: 671-675, 2014.

67. Banko AV, Lazarevic IB, Karalic DZ, Djukic VB, Cupic MD Stevanovic G and Jovanovic TP: The sequence analysis of Epstein-Barr virus EBNA1 gene: Could viral screening markers for nasopharyngeal carcinoma be identified? Med Mircobiol Immunol 208: 81-88, 2019.

68. Banko AV, Lazarevic IB, Folic MM, Djukic VB, Cirkovic AM, Karalic DZ, Cupic MD and Jovanovic TP: Characterization of the variability of Epstein-Barr virus genes in nasopharyngeal biopsies: Potential predictors for carcinoma progression. PLoS One 11: e0153498, 2016.

69. Hui EP, Ma BB, Chan KC, Chan CM, Wong CS, To KF, Chan AW, Tung SY, Ng WT, Cheng AC, et al: Clinical utility of plasma Epstein-Barr virus DNA and ERCC1 single nucleotide polymorphism in nasopharyngeal carcinoma. Cancer 121: 2720-2729, 2015.

70. Chatsirisupachai K, Palmer D, Ferreira S and de Magalhães JP: A human tissue-specific transcriptomic analysis reveals a complex relationship between aging, cancer, and cellular senescence. Aging Cell 18: e13041, 2019.

71. Sager M, Yeat NC, Pajaro-Van der Stadt S, Lin C, Ren Q and Lin J: Transcriptomics in cancer diagnostics: Developments in technology, clinical research and commercialization. Expert Rev Mol Diagn 15: 1589-1603, 2015.

72. Moor AE and Itzkovitz S: Spatial transcriptomics: Paving the way for tissue-level systems biology. Curr Poin Biotechnol 46: $126-133,2017$

73. Hayes J, Peruzzi PP and Lawler S: MicroRNAs in cancer: Biomarkers, functions and therapy. Trends Mol Med 20: 460-469, 2014.

74. Wang LJ, Chou YF, Chen PR, Su B, Hsu YC, Chang CH and Lee JW: Differential miRNA expression in repeated recurrence of nasopharyngeal carcinoma. Cancer Lett 344: 188-194, 2014.

75. Li T, Chen JX, Fu XP, Yang S, Zhang Z, Chen KH and Li Y: microRNA expression profiling of nasopharyngeal carcinoma. Oncol Rep 25: 1353-1363, 2011.

76. Lu T, Guo Q, Lin K, Chen H, Chen Y, Xu Y, Lin C, Su Y, Chen Y, Chen $\mathrm{M}$, et al: Circulating Epstein-Barr virus microRNAs BART7-3p and BART13-3p as novel biomarkers in nasopharyngeal carcinoma. Cancer Sci 111: 1711-1723, 2020.

77. Wu L, Wang J, Zhu D, Zhang S, Zhou X, Zhu W, Zhu J and He X Circulating Epstein-Barr virus microRNA profile reveals nove biomarker for nasopharyngeal carcinoma diagnosis. Cancer Biomark 27: 365-375, 2020.

78. Hirai N, Wakisaka N, Knodo S, Aga M, Moriyama-Kita M, Ueno T, Nakanishi Y, Endo K, Sugimoto H, Murono S, et al: Potential interest in circulating miR-BART17-5p as a posttreatment biomarker for prediction of recurrence in Epstein-Barr virus-related nasopharyngeal carcinoma. PLoS One 11 e0163609, 2016.

79. Zhang H, Zou X, Wu L, Zhang S, Wang T, Liu P, Zhu W and Zhu J: Identification of a 7-microRNA signature in plasma as promising biomarker for nasopharyngeal carcinoma detection. Cancer Med 9: 1230-1241, 2020.

80. Yi SJ, Liu P, Chen BL, Ou-Yang L, Xiong WM and Su JP. Circulating miR-31-5p may be a potential diagnostic biomarker in nasopharyngeal carcinoma. Neoplasma 66: 825-829, 2019.

81. Wang H, Wei X, Wu B, Su J, Tan W and Yang K: Tumor-educated platelet miR-34c-3p and miR-18a-5p as potential liquid biopsy biomarkers for nasopharyngeal carcinoma diagnosis. Cancer Manag Res 11: 3351-3360, 2019.

82. Wen W, Mai SJ, Lin HX, Zhang MY, Huang JL, Hua X, Lin C, Long ZQ, Lu ZJ, Sun XQ, et al: Identification of two microRNA signatures in whole blood as novel biomarkers for diagnosis of nasopharyngeal carcinoma. J Transl Med 17: 186, 2019.

83. Wu L, Zheng K, Yan C, Pan X, Liu Y, Liu J, Wang F, Guo W, He X Li J and Shen Y: Genome-wide study of salivary microRNAs as potential noninvasive biomarkers for detection of nasopharyngeal carcinoma. BMC Cancer 19: 843, 2019.

84. Wang M, Gu B, Chen X, Wang Y, Li P and Wang K: The function and therapeutic potential of Epstein-Barr virus-encoded MicroRNAs in cancer. Mol Ther Nucleic Acids 17: 657-668, 2019.

85. Fan C, Tang Y, Wang J, Xiong F, Guo C, Wang Y, Xiang B, Zhou M, Li X, Wu X, et al: The emerging role of Epstein-Barr virus encoded microRNAs in nasopharyngeal carcinoma. J Cancer 9: 2852-2864, 2018
86. Best MG, Sol N, Kooi I, Tannous J, Westerman BA, Rustenburg F, Schellen P, Verschueren H, Post E, Koster J, et al: RNA-Seq of tumor-educated platelets enables blood-based pan-cancer, multiclass, and molecular pathway cancer diagnostics. Cancer Cell 28: 666-676, 2015.

87. Li K, Zhu X, Li L, Ning R, Liang Z, Zeng F, Su F, Huang S, Yang $X$ and Qu S: Identification of non-invasive biomarkers for predicting the radiosensitivity of nasopharyngeal carcinoma from serum microRNAs. Sci Rep 10: 5161, 2020.

88. Wu J and Hann SS: Functions and roles of long-non-coding RNAs in human nasopharyngeal carcinoma. Cell Physiol Biochem 45: 1191-1204, 2018

89. Zhao CX, Zhu W, Ba ZQ, Xu HJ, Liu WD, Zhu B, Wang L, Song YJ, Yuan S and Ren CP: The regulatory network of nasopharyngeal carcinoma metastasis with a focus on EBV, lncRNAs and miRNAs. Am J Cancer Res 8: 2185-2209, 2018.

90. He B, Zeng J, Chao W, Chen X, Huang Y, Deng K, Huang Z, Li J, Dai M, Chen S, et al: Serum long non-coding RNAs MALAT1, AFAP1-AS1 and AL359062 as diagnostic and prognostic biomarkers for nasopharyngeal carcinoma. Oncotarget 8 : 41166-41177, 2017.

91. Yao Z, Zhang Y, Xu D, Zhou X, Peng P, Pan Z, Xiao N, Yao J and Li Z: Research progress on long non-coding RNA and radiotherapy. Med Sci Monit 25: 5757-5770, 2019.

92.Liu G, Zeng X, Wu B, Zhao J and Pan Y: RNA-Seq analysis of peripheral blood mononuclear cells reveals unique transcriptional signatures associated with radiotherapy response of nasopharyngeal carcinoma and prognosis of head and neck cancer. Cancer Biol Ther 21: 139-146, 2020.

93. Shuai M, Hong J, Huang D, Zhang X and Tian Y: Upregulation of circRNA_0000285 serves as a prognostic biomarker for nasopharyngeal carcinoma and is involved in radiosensitivity. Oncol Lett 16: 6495-6501, 2018.

94. Gosho M, Nagashima K and Sato Y: Study designs and statistical analyses for biomarker research. Sensors (Basel) 12: 8966-8986, 2012.

95. Cho WC: Mass spectrometry-based proteomics in cancer research. Expert Rev Proteomics 14: 725-727, 2017.

96. Tan HT, Lee YH and Chung MC: Cancer proteomics. Mass Spectrom Rev 31: 583-605, 2012.

97. Zhang Z, Wu S, Stenoien DL and Paša-Tolić L: High-throughput proteomics. Annu Rev Anal Chem (Palo Alto Calif) 7: 427-454, 2014.

98. Aslam B, Basit M, Nisar MA, Khurshid M and Rasool MH: Proteomics: Technologies and their applications. J Chromatogr Sci 55: 182-196, 2017.

99. EI-Sharkawy A, Al Zaidan L and Malki A: Epstein-Barr virus-associated malignancies: Roles of viral oncoproteins in carcinogenesis. Front Oncol 8: 265, 2018.

100.Zamanian Azodi M, Rezaei Tavirani M, Rezaei Tavirani M, Vafaee R and Rostami-Nejad M: Nasopharyngeal carcinoma protein interaction mapping analysis via proteomic approaches. Asian Pac J Cancer Prev 19: 845-851, 2018.

101. Cao Y: EBV based cancer prevention and therapy in nasopharyngeal carcinoma. NPJ Precis Oncol 1: 10, 2017

102. Schmidlin T, Garrigues L, Lane CS, Mulder TC, van Doorn S, Post H, de Graaf EL, Lemeer S, Heck AJ and Altelaar AF: Assessment of SRM, MRM(3), and DIA for the targeted analysis of phosphorylation dynamics in non-small cell lung cancer. Proteomics 16: 2193-2205, 2016

103. Luo Y, Mok TS, Lin X, Zhang W, Cui Y, Guo J, Chen X, Zhang T and Wang T: SWATH-based proteomics identified carbonic anhydrase 2 as a potential diagnosis biomarker for nasopharyngeal carcinoma. Sci Rep 7: 41191, 2017.

104. Nguyen EV, Centenera MM, Moldovan M, Das R, Irani S, Vincent $\mathrm{AD}$, Chan $\mathrm{H}$, Horvath LG, Lynn DJ, Daly RJ and Butler LM: Identification of novel response and predictive biomarkers to Hsp90 inhibitors through proteomic profiling of patient-derived prostate tumor explants. Mol Cell Proteomics 17: 1470-1486, 2018.

105. Nakamura K, Hirayama-Kurogi M, Ito S, Kuno T, Yoneyama T, Obuchi W, Terasaki T and Ohtsuki S: Large-scale multiplex absolute protein quantification of drug-metabolizing enzymes and transporters in human intestine, liver, and kidney microsomes by SWATH-MS: Comparison with MRM/SRM and HR-MRM/PRM. Proteomics 16: 2106-2117, 2016.

106. Sun X, Chen Y, Tan J and Qi X: Serum IRS-1 acts as a novel biomarker for diagnosis in patients with nasopharyngeal carcinoma. Int J Clin Exp Pathol 11: 3685-3690, 2018. 
107. Coghill AE, Pfeiffer RM, Proietti C, Hus WL, Chien YC, Lekiffre L, Krause L, Teng A, Pablo J, Yu KJ, et al: Identification of a novel, EBV-based antibody risk stratification signature for early detection of nasopharyngeal carcinoma in Taiwan. Clin Cancer Res 24:1305-1314, 2018.

108. Lin LH, Xu YW, Huang LS, Hong CQ, Zhai TT, Liao LD, Lin WJ, Xu LY, Zhang K, Li EM and Peng YH: Serum proteomic-based analysis identifying autoantibodies against PRDX2 and PRDX3 as potential diagnostic biomarkers in nasopharyngeal carcinoma. Clin Proteomics 14: 6, 2017.

109. Gong D, Li Z, Ding R, Cheng M, Huang H, Liu A, Kang M, $\mathrm{He} \mathrm{H}, \mathrm{Xu} \mathrm{Y}$, Shao J, et al: Extensive serum biomarker analysis in patients with nasopharyngeal carcinoma. Cytokine 118: 107-114, 2019

110. Meng H, Zhu X, Li L, Liang Z, Li X, Pan X, Zeng F and Qu S: Identification of CALM as the potential serum biomarker for predicting the recurrence of nasopharyngeal carcinoma using a mass spectrometry based comparative proteomic approach. Int J Mol Med 40: 1152-1164, 2017

111. Manes NP and Nita-Lazar A: Application of targeted mass spectrometry in bottom-up proteomics for systems biology research. J Proteomics 189: 75-90, 2018.

112. Sun Y, Guo W, Bai Y, Ge M, Hu C, Wu S, Hao J, Gao M, Pan J, Dong P, et al: Neoadjuvant dose-modified docetaxel in squamous cell carcinoma of the head and neck: A phase 3 study. Oral Dis 26: 285-294, 2020.

113. Monti C, Zilocchi M, Colugnat I and Alberio T: Proteomics turns functional. J Proteomics 198:36-44, 2019.

114. Xiao Z and Chen Z: Deciphering nasopharyngeal carcinoma pathogenesis via proteomics. Expert Rev Proteomics 16: 475-485, 2019.

115. Wang X: Cancer moonshot 2020: A new march of clinical and translational medicine. Clin Transl Med 5: 11, 2016.

116. Fiore LD, Rodriguez H and Shriver CD: Collaboration to accelerate proteogenomics cancer care: The department of veterans affairs, department of defense, and the national cancer institute's applied proteogenomics organizational learning and outcomes (APOLLO) network. Clin Pharmacol Ther 101: 619-621, 2017.

117. Rinschen MM, Ivanisevic J, Giera M and Siuzdak G: Identification of bioactive metabolites using activity metabolomics. Nat Rev Mol Cell Biol 20: 353-367, 2019.

118. Guijas C, Montenegro-Burke JR, Warth B, Spilker ME and Siuzdak G: Metabolomics activity screening for identifying metabolites that modulate phenotype. Nat Biotechnol 36 316-320, 2018

119. Schrimpe-Rutledge AC, Codreanu SG, Sherrod SD and McLean JA: Untargeted metabolomics strategies-challenges and emerging directions. J Am Soc Mass Spectrom 27: 1897-1905, 2016

120. Li X, Wenes M, Romero P, Huang SC, Fendt SM and Ho PC: Navigating metabolic pathways to enhance antitumour immunity and immunotherapy. Nat Rev Clin Oncol 16: 425-441, 2019

121. Yang M, Soga T and Pollard PJ: Oncometabolites: Linking altered metabolism with cancer. J Clin Invest 123: 3652-3658, 2013

122. Li S, Fu L, Tian T, Deng L, Li H, Xia W and Gong Q: Disrupting SOD1 activity inhibits cell growth and enhances lipid accumulation in nasopharyngeal carcinoma. Cell Commun Signal 16: 28,2018

123. Tan Z, Xiao L, Tang M, Bai F, Li J, Li L, Shi F, Li N, Li Y, Du Q, et al: Targeting CPT1A-mediated fatty acid oxidation sensitizes nasopharyngeal carcinoma to radiation therapy. Theranostics 8: 2329-2347, 2018.

124. Tang F, Xie C, Huang D, Wu Y, Zeng M, Yi L, Wang L, Mei W, Cao Y and Sun L: Novel potential markers of nasopharyngea carcinoma for diagnosis and therapy. Clin Biochem 44: 711-718, 2011

125. Yi L, Dong N, Shi S, Deng B, Yun Y, Yi Z and Zhang Y: Metabolomic identification of novel biomarkers of nasopharyngeal carcinoma. RSC Adv 4: 59094-59101, 2014.

126. Luo MS, Huang GJ and Liu HB: Oncologic outcomes of IMRT versus CRT for nasopharyngeal carcinoma: A meta-analysis. Medicine (Baltimore) 98: e15951, 2019.
127. Carayol M, Leitzmann MF, Ferrari P, Zamora-Ros R, Achaintre D, Stepien M, Schmidt JA, Travis RC, Overvad K, Tjønneland A, et al: Blood metabolic signatures of body mass index: A targeted metabolomics study in the EPIC cohort. J Proteome Res 16: 3137-3146, 2017.

128. Xu J, Li J, Zhang R, He J, Chen Y, Bi N, Song Y, Wang L, Zhan Q and Abliz Z: Development of a metabolic pathway-based pseudo-targeted metabolomics method using liquid chromatography coupled with mass spectrometry. Talanta 192: 160-168, 2019.

129. Allinson JL: Clinical biomarker validation. Bioanalysis 10: 957-968, 2018.

130. Rhomdhoni AC, Kurniawan P and Hidayati T: Correlation between superoxide dismutase serum level alteration with neck metastatic tumor post cisplatin-paclitaxel chemotherapy response in nasopharyngeal carcinoma patients. Indian J Otolaryngol Head Neck Surg 71 (Suppl 1): S643-S646, 2019.

131. Jelonek K, Krzywon A, Jablonska P, Slominska EM, Smolenski RT, Polanska J, Rutkowski T, Mrochem-Kwarciak J, Skladowki K and Widlak P: Systemic effects of radiotherapy and concurrent chemo-radiotherapy in head and neck cancer patients-comparison of serum metabolome profiles. Metabolites 10: 60, 2020

132. Geyer PE, Voytik E, Treit PV, Doll S, Kleinhempel A, Niu L, Müller JB, Buchholtz ML, Bader JM, Teupser D, et al: Plasma proteome profiling to detect and avoid sample-related biases in biomarker studies. EMBO Mol Med 11: e10427, 2019.

133. Dufourd T, Robil N, Mallet D, Carcenac C, Boulet S, Brishoual S, Rabois E, Houeto JL, de la Grange P and Carnicella S: Plasma or serum? A qualitative study on rodents and humans using high-throughput microRNA sequencing for circulating biomarkers. Biol Methods Protoc 4: bpz006, 2019.

134. Lippi G,Banfi G,Buttarello M,CeriottiF,Daves M,Dolci A,CaputoM, Giavarina D, Montagnana M, Miconi V, et al: Recommendations for detection and management of unsuitable samples in clinical laboratories. Clin Chem Lab Med 45: 728-736, 2007.

135. Lam WKJ, Jiang P, Chan KCA, Cheng SH, Zhang H, Peng W, Tse OYO, Tong YK, Gai W, Zee BCY, et al: Sequencing-based counting and size profiling of plasma Epstein-Barr virus DNA enhance population screening of nasopharyngeal carcinoma. Proc Natl Acad Sci USA 115: E5115-E5124, 2018.

136. Elliott P and Peakman TC; UK Biobank: The UK biobank sample handling and storage protocol for the collection, processing and archiving of human blood and urine. Int $\mathbf{J}$ Epidemiol 37: 234-244, 2008

137. Gautam A, Donohue D, Hoke A, Miller SA, Srinivasan S, Sowe B, Detwiler L, Lynch J, Levangie M, Hammamieh R and Jett M: Investigating gene expression profiles of whole blood and peripheral blood mononuclear cells using multiple collection and processing methods. PLoS One 14: e0225137, 2019.

138. Leidinger P, Backes C, Rheinheimer S, Keller A and Meese E: Towards clinical applications of blood-borne miRNA signatures: The influence of the anticoagulant EDTA on miRNA abundance. PLoS One 10: e0143321, 2015.

139. Mathé E, Hays JL, Stover DG and Chen JL: The omics revolution continues: The maturation of high-throughput biological data sources. Yearb Med Inform 27: 211-222, 2018.

140. Hasin Y, Seldin M and Lusis A: Multi-omics Approaches to Disease. Genome Biol 18: 83, 2017.

141. Chakraborty S, Hosen MI, Ahmed M and Shekhar HU: Onco-multi-OMICS approach: A new frontier in cancer research. Biomed Res Int 2018: 9836256, 2018.

142. Nicora G, Vitali F, Dagliati A, Geifman N and Bellazzi R: Integrated multi-omics analyses in oncology: A review of machine learning methods and tools. Front Oncol 10: 1030, 2020

143. Olivier M, Asmis R, Hawkins GA, Howard TD and Cox LA The need for multi-omics biomarker signatures in precision medicine. Int J Mol Sci 20: 4781, 2019.

This work is licensed under a Creative Commons Attribution-NonCommercial-NoDerivatives 4.0 International (CC BY-NC-ND 4.0) License. 